\title{
From Genome Variation to Molecular Mechanisms: What we Have Learned From Yeast Mitochondrial Genomes?
}

\author{
Weilong Hao* \\ Department of Biological Sciences, Wayne State University, Detroit, MI, United States
}

OPEN ACCESS

Edited by:

Georg Hausner,

University of Manitoba, Canada

Reviewed by:

Heather Fiumera,

Binghamton University, United States

Feng Ling,

RIKEN, Japan

Hsin-Han Lee,

Academia Sinica, Taiwan

*Correspondence:

Weilong HaO

haow@wayne.edu

Specialty section:

This article was submitted to

Evolutionary and Genomic

Microbiology

a section of the journal

Frontiers in Microbiology

Received: 01 November 2021

Accepted: 03 January 2022

Published: 20 January 2022

Citation:

Hao W (2022) From Genome Variation to Molecular Mechanisms: What we Have Learned From Yeast

Mitochondrial Genomes?

Front. Microbiol. 13:806575. doi: 10.3389/fmicb.2022.806575
Analysis of genome variation provides insights into mechanisms in genome evolution. This is increasingly appreciated with the rapid growth of genomic data. Mitochondrial genomes (mitogenomes) are well known to vary substantially in many genomic aspects, such as genome size, sequence context, nucleotide base composition and substitution rate. Such substantial variation makes mitogenomes an excellent model system to study the mechanisms dictating mitogenome variation. Recent sequencing efforts have not only covered a rich number of yeast species but also generated genomes from abundant strains within the same species. The rich yeast genomic data have enabled detailed investigation from genome variation into molecular mechanisms in genome evolution. This mini-review highlights some recent progresses in yeast mitogenome studies.

Keywords: mutation, recombination, repeat, mobile introns, gene conversion, GC-content

\section{INTRONDUCTION}

Mitochondrial genomes (mitogenomes) originated from an alpha-proteobacterium via endosymbiosis (Lang et al., 1999), and have adopted radically different shapes, sizes, and organizations (Burger et al., 2003; Shao et al., 2009; Sloan et al., 2012; Smith et al., 2012). The great variation of mitogenome diversity and complexity has revolutionized our view of genome evolution and facilitated development of new evolutionary theories (Bazin et al., 2006; Lynch et al., 2006; Whitney and Garland, 2010; Sloan et al., 2012; Christensen, 2013). The excitement of mitogenomes inspired more sequencing projects, and perhaps more importantly, many mindful and in-depth comparative genomics studies (Smith, 2015). These efforts continue to push the boundaries of our understanding in genome evolution.

Mitogenomes are highly variable among yeast species. Mitogenomes show substantial differences in genome size and organization, GC-content, mutation rates, and recombination frequencies. As mutation is the fundamental source of genetic variation, mitogenome differences provide important insights into the underlying mechanisms in the mutation processes. The budding yeast Saccharomyces cerevisiae is among the best-studied model organisms, an abundant number of mitogenomes have been sequenced for S. cerevisiae (Strope et al., 2015; De Chiara et al., 2020) and related species (Freel et al., 2014; Nguyen et al., 2020b). The abundant genomic data have allowed comparative analyses of mitogenomes among closely related yeast species and also intraspecific strains within a species. Detailed variations among closely related mitogenomes become uniquely informative to help identify molecular mechanisms driving the genomic changes.

\section{MITOCHONDRIAL DNA DELETION}

The mitochondrial DNAs (mtDNAs) of related yeast strains is well known to show hypervariability in sequence [reviewed in Borst and Grivell (1978)]. Studies of hypervariable yeast mitogenomes 
have led to discoveries of new molecular and cellular mechanisms (Dujon, 2020). Yeast spontaneously loses mtDNAs and develops respiratory-incompetent petite colonies ( $\mathrm{rho}^{-}$) (Whittaker et al., 1972). Petite colonies arise naturally under normal growth conditions, but the frequency of petite formation varies among strains. In S. cerevisiae, the frequency of developing spontaneous petite colonies varies by about 100-fold between laboratory strains versus natural isolates (Dimitrov et al., 2009). The formation of petite colonies, or petite-positive trait, has been associated with whole-genome duplication of the nuclear genome (Piskur, 2001), which provides the basis for neofunctionalization. A survey in more than one hundred strains, however, shows that the petite positive trait is throughout the Saccharomycetaceae family, much beyond the whole genome duplication species (Fekete et al., 2007). Some deletions in yeast mtDNAs are mediated by gene conversion between GC cluster repeats (Weiller et al., 1991), which are characterized by high GC content and palindromic structure (Yin et al., 1981) (also described below). Petite formation, as a genetic trait, has been studied in genome-wide association studies to identify the associated genes (Dimitrov et al., 2009). The different frequencies in petite formation among strains have been associated with genetic variation in at least four nuclear-encoded genes, the mtDNA polymerase (MIP1) (Baruffini et al., 2007a) and other less-well studied SAL1, CAT5, and MKT1 genes (Dimitrov et al., 2009).

\section{MOVEMENT OF MITOCHONDRIAL INTRONS}

Genetic crosses of different mitochondrial genotypes have been conducted in S. cerevisiae to understand yeast mitochondrial inheritance and genetics (Wilkie and Thomas, 1973). Mitochondrial markers are inherited in a non-mendelian manner (Jacquier and Dujon, 1985). A well-studied example is the omega ( $\omega$ ) locus in 21S rRNA (Butow, 1985), in which the $\omega+$ strains can transfer the whole intron unidirectionally to an intron-less locus (Dujon et al., 1974). The $\omega$ locus belongs to group I intron, a class of self-catalytic ribozymes that often encode a homing endonuclease gene (HEG) (Perlman and Butow, 1989). The self-spicing intron and intron-encoded endonuclease play an important role in driving intron invasion and mobility (Dujon, 1989). The mobility and invasion of the $\omega$ intron can go beyond the species boundary. The phylogeny of $\omega$-HEG is significantly different from that of the host $21 \mathrm{~S}$ rRNA (Goddard and Burt, 1999), suggesting cross-species horizontal transfer of $\omega$-HEG. Given the highly invasive nature of the $\omega$ intron, one would expect most yeast strains to be $\omega+$. Yet, many yeast strains remain $\omega-$. Goddard and Burt (1999) provided a framework to explain the sporadic distribution of the $\omega$ intron; after invasion, the omega-HEGs undergo rapid degeneration and loss, followed by reinvasion. This process is coined as the Goddard-Burt life cycle by Mukhopadhyay and Hausner (2021). The actual life cycle of introns is likely much more complex than cyclical invasion, degeneration, loss and then reinvasion.

In the Saccharomycetaceae family, mitochondrial introns are found in three genes, cox $1, c o b$, and $21 \mathrm{~S}$ rRNA, in total 17 intron positions (Wu et al., 2015). Each intron has a unique distribution pattern, and intron content often varies substantially even among small numbers of conspecific strains within species. Except the cox 1 il intron, all other 16 introns are sporadically distributed. The evolutionary turnover rates of gain and loss among these introns were quantitatively measured (Wu et al., 2015). The high-mobility introns documented in genetic crosses do not necessarily have faster turnover rates than low-mobility introns. The cox1 i1 intron is currently only found in S. cerevisiae strains, with a high rate of intron insertion, it will not be surprising if the cox $1 \mathrm{i} 1$ intron is present in some upcoming non-S. cerevisiae mitogenomes. Furthermore, phylogenetically mosaic sequences are evident in both introns and HEGs (Wu and Hao, 2014). Thus, intron and its encoded HEG do not always transmit together as a unit. These findings support that gene conversion between the donor and recipient sequences can take place at both the gene and intragenic levels and lead to insertion or deletion of the adjacent HEG/intron content (Wu and Hao, 2014).

\section{ELEVATED SEQUENCE EVOLUTION NEAR MOBILE INTRONS}

The distribution of mutation along DNA sequences is not uniform, and yeast mitogenomes are no exception. The recent availability of abundant yeast population genomic data makes it possible to examine genetic diversity along mitochondrial genes. One striking finding is the increased density of single nucleotide polymorphisms (SNP) in exon regions approaching intron boundaries (Repar and Warnecke, 2017). Although intron mobility is recognized to play a critical role in driving the sequence diversity of host genes, the underlying mechanisms cannot be easily identified. There are two possible mechanisms that can increase SNP density in exons (Repar and Warnecke, 2017). First, diverse exonic sequences are gene conversion tracts, or known as co-conversion tracts, acquired from distantly related species. Horizontal transfer of introns and co-conversion tracts has been well documented in plant mitogenomes (Cho and Palmer, 1999). Since most plant mitogenomes have exceptionally low mutation rates, shared long co-conversion tracts among distantly related intron-containing sequence can be easily and convincingly identified (Sanchez-Puerta et al., 2011). Similar to plant mitochondrial introns, yeast mitochondrial introns also undergo horizontal transfer (Wu et al., 2015) and gene conversion at intragenic level (Wu and Hao, 2014). The relative high sequence divergence among yeast mitogenomes makes it challenging to accurately identify the donor species of the diverse co-conversion tracts. Alternatively, the flanking regions of each intron insert site are mutation hotspots because of endonuclease activity and subsequent error-prone repair. Yeast mitogenomes contain stand-alone HEGs, which are not associate with mitochondrial introns. A strong association is evident between the presence of a stand-alone endonuclease gene and high sequence diversity at the end of the endonucleaseadjacent gene (Wu and Hao, 2019). This finding is consistent with the notion that the recognition sites of endonuclease are mutation hotspots. 


\section{THE REBIRTH OF GC-CLUSTERS}

The var1 gene (or called rps3 in non-yeast fungi) is another well-studied example of unidirectional inheritance in S. cerevisiae (Strausberg and Butow, 1981). The varl gene is polymorphic, and different forms of the varl gene differ by in-frame insertions of short GC-rich palindromic cluster (GC-cluster) in the coding region (Hudspeth et al., 1984). The terminal sequences of most GC clusters are repeats and different GC clusters can share the same terminal repeats. For instance, AG dinucleotide and TAG trinucleotide repeats are common among GC-clusters in S. cerevisiae (Weiller et al., 1989). The terminal repeats are regarded as target-site duplication, and GC-clusters have been suggested to bear ribozyme activity, which catalyzes self-cleavage and ligation reactions (Weiller et al., 1989; Lang et al., 2014). GC-cluster sequences rapidly accumulate substitutions especially in the loop regions, and also undergo dynamic merger and shuffling to form new GCclusters (Wu and Hao, 2015). Changes at nucleotide bases as well as sequence structure result in highly variable GC cluster sequences among different yeast mitogenomes. GC-clusters are most often found in intergenic regions, but many of them are transcribed into RNAs (Wu and Hao, 2015). All these support the notion that GC clusters are transposable elements. GCclusters can also be found in protein-coding regions. In yeast Magnusiomyces capitatus (in the Dipodascaceae family), GCclusters inserted in protein-coding regions are transcribed in mRNAs, but the GC-cluster region in mRNA gets bypassed (or ignored) during translation (Lang et al., 2014). GC clusters have been suggested as recombination hotspots in mitogenomes (Dieckmann and Gandy, 1987). GC cluster-mediated gene conversion can insert or delete large genomic fragments (Weiller et al., 1991), which ultimately lead to alteration of genome size. GC clusters have been suggested to induce long ATrich sequences into the Nakaseomyces bacillisporus mitogenome (Bouchier et al., 2009).

\section{MITOCHONDRIAL DNA RECOMBINATION}

Pioneer studies on mtDNA recombination through mating between $S$. cerevisiae strains can be traced back to the early 1970s (Kleese et al., 1972; Shannon et al., 1972). Recent large-scale genomic survey confirms frequent mtDNA recombination in natural $S$. cerevisiae populations (De Chiara et al., 2020). Surprisingly, yeasts were not among the organisms in early discoveries of mtDNA recombination between different species, as shown in plants (Hao et al., 2010; Mower et al., 2010). Subsequent analysis on closely related yeast mitogenomes found extensive recombination throughout the mitogenome between yeast species ( $\mathrm{Wu}$ et al., 2015). There are two important identified issues in assessing the extent of mtDNA recombination events. (1) Accurate detection of mtDNA recombination relies on the abundance of closely related mitogenomes, preferably, an abundant number of intraspecific mitogenomes from several related species. (2) Many mtDNA recombinant events are fine-scale and often overlooked when using whole genes as the unit of phylogenetic analysis.

Fritsch et al. (2014) have constructed the genome-wide map of mtDNA recombination events in S. cerevisiae and found that recombinant hotspots are preferentially localized in intergenic and intronic regions. They further investigated the impact of individual depletion of four genes [namely Ntg1 (Ling et al., 2007), Mgt1 (Lockshon et al., 1995), MHR1, and Din7 (Ling et al., 2013)] previously associated with mtDNA recombination. The deletion of Ntg1, Mgt1, and MHR1 had little influence on mtDNA recombination hotspots, and the deletion of $\operatorname{Din} 7$ resulted primarily in DNA degradation. These findings hint that the study of only nuclear-encoded genes is unlikely to achieve a complete understanding of the localization of recombination hotspots along the yeast mitogenome. Given the dynamic nature of mitochondrial -encoded sequences, such as introns and GC-clusters, a plausible alternative could be that mtDNA recombination hotspots are primarily driven by the sequence features of the mitogenome. Future studies are needed to test this hypothesis.

Mitochondrial DNA recombination can impact mitochondrial function in different ways. As described above (on GC-clusters), Non-allelic mtDNA recombination can lead to deletion and insertion of sequences. Allelic mtDNA recombination, on the other hand, can prevent mtDNA deletion and repair mitogenome damage (Ling et al., 2019). Mitochondrial recombination introduces rapid sequence changes, and could have a significant functional impact on the host. There are direct functional impacts of the introduced mito-genotype, effects of the altered mito-nuclear interaction, and effects of the interaction between the introduced mito-loci and native mitoloci (mito-mito interaction) (Wolters et al., 2018). Mitochondrial recombination can enhance phenotypic variation among diploid hybrids, and facilitate the phenotypic differentiation of hybrid species (Leducq et al., 2017). Given the prevalence of yeast mtDNA recombination and even across different species, much phenotypic diversity in yeast could have resulted from mitochondrial recombination.

\section{YEAST MITOGENOMES ARE HIGHLY VARIABLE IN SIZE}

In the Saccharomycetaceae family, the smallest and largest mitogenomes belong in the same genus Nakaseomyces (Figure 1). The mitogenome in Candida glabrata is $20.1 \mathrm{~kb}$ (Koszul et al., 2003), while the mitogenome in Nakaseomyces bacillisporus is over five-time larger, at 107.1 Kb (Bouchier et al., 2009). In contrast, the sizes of nuclear genomes among the Nakaseomyces species are remarkably similar, ranging from 10.2 to $12.3 \mathrm{Mb}$ (Gabaldon et al., 2013). In the sister family Saccharomycodaceae, Hanseniaspora uvarum has a linear mitogenome at $11.1 \mathrm{~kb}$ (Pramateftaki et al., 2006), and Saccharomycodes ludwigii has a circular mitogenome at $69.0 \mathrm{~kb}$ (Nguyen et al., 2020b). Mitogenome sizes also vary among strains within the same yeast species. Among the 109 S. cerevisiae mitogenomes examined in 


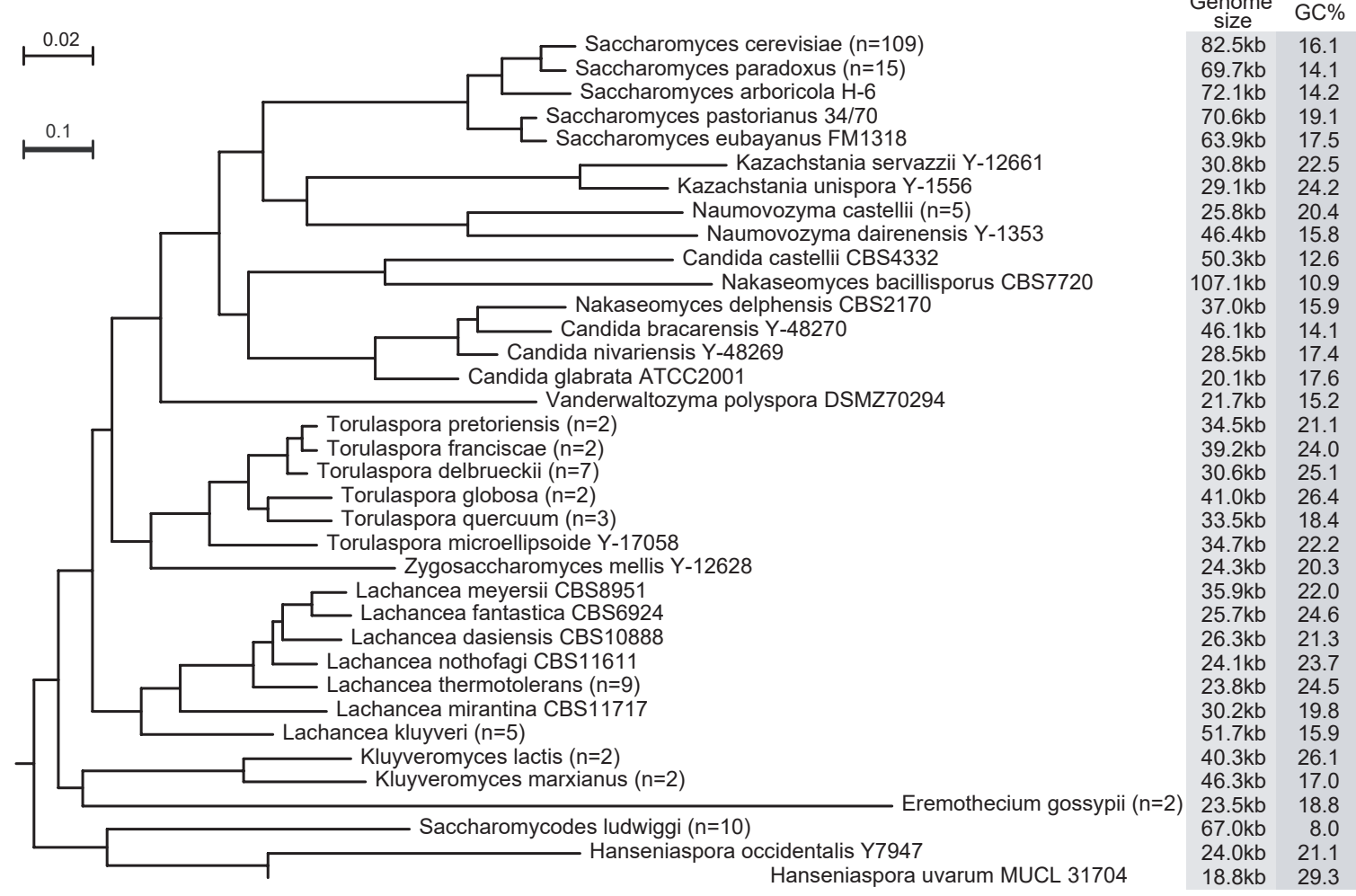

FIGURE 1 | Variation in size, sequence divergence, and GC-content of mtDNAs of 36 yeast species. The phylogenetic tree is based on nucleotide sequences of seven core mitochondrial genes (cob, cox1, cox2, cox3, atp6, atp8, and atp9). The very long branch leading to Hanseniaspora uvarum is shown by a thickened line at fivefold reduced scale. If two or more strains are analyzed in a species, the averages are shown for genome size and GC\% (strain number in parentheses). Otherwise, the strain names are shown. Details of all strain names in the Saccharomycetaceae family are in Xiao et al. (2017), and the Saccharomycodes /udwigii strains are in Nguyen et al. (2020b).

Xiao et al. (2017), and their genome sizes range from 74.2 to $92.2 \mathrm{~kb}$, The fast increasing number of yeast mitogenomes will only expand the range in mitogenome size difference.

\section{STABLE GENE CONTENT IN YEAST MITOGENOMES}

Despite the variable sizes, genes encoded in yeast mitogenomes are remarkably stable. Unlike mitogenomes in many other fungal species, none of the mitogenomes in Saccharomycetaceae and Saccharomycodaceae encode the respiratory-chain NADH dehydrogenase (complex I) (Dujon, 2010; Freel et al., 2014). The loss of complex I in yeasts is generally believed as a result of adaptation to fermentative lifestyles, where complex I is not essential (Schikora-Tamarit et al., 2021). All complete mitogenomes in Saccharomycetaceae encode eight protein genes, seven respiratory-chain protein genes (atp6, atp8, atp9, cob, $\operatorname{cox} 1, \cos 2$, and $\operatorname{cox} 3$ ) and one ribosomal protein gene var1, SSU and LSU rRNAs, and 22-24 tRNAs. This is in contrast with a recent report that "no gene is universally conserved in fungal mitogenomes" (Fonseca et al., 2021). Although it is inevitable that fewer mitochondrial genes are shared when more diverse lineages are included (Roger et al., 2017), the variation of mitogenome sequence quality could have been an important reason for the discrepancy on gene conservation among yeast mitogenomes. For instance, the two $S$. cerevisiae mitogenomes (accessions: CM002421 and CP046458) reportedly missing atp6 by Fonseca et al. (2021) are 22,149 bp (including 13,247 Ns for gaps) and 49,451 bp (517 Ns) in length, respectively, much shorter than the average $82.5 \mathrm{~Kb}$ [ranging from 74.2 to $92.2 \mathrm{~kb}$ in Xiao et al. (2017)] of $S$. cerevisiae mitogenomes (Figure 1). The Saccharomycodaceae family has a sole case of gene loss involving a protein gene. That is the var1 gene absent from Hanseniaspora uvarum (Pramateftaki et al., 2006). This is likely a lineagespecific gene loss, as var1 is present in Saccharomycodes ludwigii (Nguyen et al., 2020b), a related species within the Saccharomycodaceae family.

\section{FACTORS DRIVING MITOGENOME SIZE VARIATION}

Mobile introns and variable intergenic regions are known factors driving variation of yeast mitogenome architecture (Bouchier et al., 2009; Freel et al., 2014). Ironically, the mitogenome in Nakaseomyces bacillisporus at $107 \mathrm{~kb}$ contains no intron, while 
mitogenomes in Candida glabrata at around $20 \mathrm{~kb}$ contain 34 introns in at least four intron-distribution patterns [(Koszul et al., 2003), and unpublished observation]. This irony can be solved by separate analyses at different time scales (Xiao et al., 2017). When intraspecific mitogenomes (within the same species) are compared, intron sequences show the highest variance in length and significantly overrepresented in large mitogenomes. When interspecific mitogenomes (among different species) are compared, tandem repeats show the highest variance in sequence length and significantly overrepresented in large mitogenomes. In other words, the rapid turnover of mobile introns can significantly impact genome size, but the number of available introns insertion sites are limited; while expansion and contraction of repeats may cause only subtle change per event, but they take place persistently with little space limit.

\section{ORIGIN OF MITOGENOME SIZE}

The question on whether genome size is under selective constraint has been a subject of debate (Lynch and Conery, 2003; Whitney and Garland, 2010; Shtolz and Mishmar, 2019). The mutational burden hypothesis $(\mathrm{MBH})$ was postulated to explain the origin of organellar genome size (Lynch et al., 2006). Introns and intergenic DNAs in mitogenomes are genetic liability, as they are targets for deleterious and potentially lethal mutations (Lynch et al., 2006). Following $\mathrm{MBH}$, introns and intergenic DNAs tend to accumulate when natural selection is less efficient at purging hazardous non-coding DNA. Using the ratio of nonsynonymous over synonymous $\mathrm{Ka} / \mathrm{Ks}$ rates as a proxy for the level of genetic drift, a significant positive correlation was evident between the genome-wide $\mathrm{Ka} / \mathrm{Ks}$ ratios and mitogenome sizes among seven yeast species with sufficient intraspecific diversity (Xiao et al., 2017). This finding is consistent with the notion that introns, GC-clusters, and repeats in yeast mitogenomes are mostly deleterious (Bernardi, 2005; Wu and Hao, 2014, 2015). Hanseniaspora uvarum has accelerated sequence evolution compared with related species (Figure 1) and has a small genome size, which is consistent with the $\mathrm{MBH}$ hypothesis. Genetic drift still faces challenges to explain mitogenome size variation in many yeast species. For example, significant relaxation of mitochondrial functions has been documented after wholegenome duplication (Jiang et al., 2008), yet many post-wholegenome duplication species (including Candida glabrata) have compact mitogenomes. Unfortunately, many yeast species either lack intraspecific genomic data or suffer insufficient sequence diversity, making it impossible to estimate the degree of genetic drift. To address this issue, extended efforts are needed to sample and sequence an abundant number of intraspecific strains in a broad range of yeast species.

\section{EXTREME GENOME-WIDE G + C CONTENT}

Even though yeast mitogenomes are overall AT-rich, their G + Ccontents vary greatly. GC-content of the Hanseniaspora uvarum mitogenome is 29.3\% (Pramateftaki et al., 2006), while the most AT-rich mitogenome in Saccharomycodes ludwigii is at $7.6 \%$ G + C (Nguyen et al., 2020b). Generally speaking, mutation is nearly universally biased from $\mathrm{C} / \mathrm{G}$ to $\mathrm{T} / \mathrm{A}$ (Hershberg and Petrov, 2010), and mutation rates are often higher at $C / G$ nucleotides than at A/T nucleotides (Zhu et al., 2014). Alterations of GC content have been shown to impact mutation and recombination rates (Kiktev et al., 2018). The mitogenomes with extreme base composition offer important and unique insights into the mechanisms governing mutation processes (Gardner et al., 2002; McCutcheon and Moran, 2010; Smith et al., 2011; Su et al., 2019). Comparative genomics of the 10 extreme AT-rich mitogenomes in Saccharomycodes ludwigii (Nguyen et al., 2020b) found a strong mutation bias toward $\mathrm{A} / \mathrm{T}$, but the expected equilibrium $\mathrm{G}+\mathrm{C}$ content under mutation pressure alone is still higher than observed $\mathrm{G}+\mathrm{C}$ content. Interestingly, mitogenomes in Saccharomycodes ludwigii undergo frequent recombination, a genetic process that normally increases $\mathrm{G}+\mathrm{C}$ content by GC-biased gene conversion (Pessia et al., 2012). These findings suggest other mechanisms alongside with AT-biased mutation operating to increase A/T in Saccharomycodes ludwigii. Another important, but perhaps underappreciated finding is the prevalence of indel mutations in yeast mitogenomes (Xiao et al., 2017). Indel mutations contribute much more to genomic variation among closely related mitogenomes than nucleotide substitutions. Further studies are needed to investigate the molecular mechanisms driving indel mutations and quantitatively model the evolutionary process of indel mutations.

\section{VARIABLE EVOLUTIONARY RATES AMONG YEAST MITOGENOMES}

Yeast mitogenomes show variable evolutionary rates. The branch leading to Hanseniaspora uvarum is at least five times longer than the branch leading to its sister species. Similarly, the branch leading to a plant pathogen Eremothecium gossypii is at least three times longer than the branch leading to the related Kluyveromyces species (Figure 1). Spontaneous mitochondrial mutation rates have been measured in several yeast species. The mitochondrial base-substitution mutation (BSM) rates are all higher than their corresponding nuclear BSM rates. The mitochondrial BSM rates in S. cerevisiae range from $4.47 \times 10^{-10}$ (Sharp et al., 2018) to $122.3 \times 10^{-10}$ per site per cell division (Lynch et al., 2008). Please also note that the $S$. cerevisiae FY10 strain (isogenic to S288c) used in the Lynch et al. (2008) study contains a single non-synonymous mutation in the mtDNA polymerase (MIP1) linked to reduced fidelity of mtDNA replication (Baruffini et al., 2007b). Hanseniaspora uvarum does have a higher mitochondrial $\mathrm{BSM}$ rate than other two Hanseniaspora species, at $13.1 \times 10^{-10}$ mutations per site per cell division, compared with $5.94 \times 10^{-10}$ mutations per site per cell division in Hanseniaspora valbyensis, and $3.65 \times 10^{-10}$ mutations per site per cell division in Hanseniaspora osmophila (Nguyen et al., 2020a). The measured spontaneous mutation rates will also help us estimate the effective population size for each species following equation 
$\pi_{\text {silent }}=2 \mathrm{~N}_{e} \mu$, where $\pi_{\text {silent }}$ is nucleotide diversity at silent sites, $\mathrm{N}_{e}$ is effective population size, $\mu$ is mutation rate (Lynch, 2006). Precise estimation of effective population size for mtDNA holds the key to understanding the significance of mtDNA recombination at the population level. To achieve this, it is critical to obtain both intraspecific diversity and spontaneous mutation rate for a variety of yeast species. Extra attention must also be paid to accumulate sufficient number of mitochondrial mutations due to the small mitogenome size relative to the nuclear genome size.

\section{MOVING FORWARD}

Yeast mitogenomes are highly diverse ranging from fast-evolving compact mitogenomes (similar to animal mitogenomes) to slow-evolving mitogenomes inflated by large introns, repeats and non-coding sequences (similar to plant mitogenomes). The fast-growing yeast mitogenome data have allowed us to begin to identify mechanisms driving genome diversity. Specific efforts are needed to sequence and study an abundant number of intraspecific strains from closely related species for

\section{REFERENCES}

Baruffini, E., Ferrero, I., and Foury, F. (2007a). Mitochondrial DNA defects in Saccharomyces cerevisiae caused by functional interactions between DNA polymerase gamma mutations associated with disease in human. Biochim. Biophys. Acta 1772, 1225-1235. doi: 10.1016/j.bbadis.2007.10.002

Baruffini, E., Lodi, T., Dallabona, C., and Foury, F. (2007b). A single nucleotide polymorphism in the DNA polymerase gamma gene of Saccharomyces cerevisiae laboratory strains is responsible for increased mitochondrial DNA mutability. Genetics 177, 1227-1231. doi: 10.1534/genetics.107.079293

Bazin, E., Glemin, S., and Galtier, N. (2006). Population size does not influence mitochondrial genetic diversity in animals. Science 312, 570-572. doi: 10.1126/ science. 1122033

Bernardi, G. (2005). Lessons from a small, dispensable genome: the mitochondrial genome of yeast. Gene 354, 189-200. doi: 10.1016/j.gene.2005.03.024

Borst, P., and Grivell, L. A. (1978). The mitochondrial genome of yeast. Cell 15, 705-723. doi: 10.1016/0092-8674(78)90257-x

Bouchier, C., Ma, L., Creno, S., Dujon, B., and Fairhead, C. (2009). Complete mitochondrial genome sequences of three Nakaseomyces species reveal invasion by palindromic GC clusters and considerable size expansion. FEMS Yeast Res. 9, 1283-1292. doi: 10.1111/j.1567-1364.2009.00551.x

Burger, G., Gray, M. W., and Lang, B. F. (2003). Mitochondrial genomes: anything goes. Trends Genet. 19, 709-716.

Butow, R. A. (1985). Nonreciprocal exchanges in the yeast mitochondrial genome. Trends Genet. 1, 81-84.

Cho, Y., and Palmer, J. D. (1999). Multiple acquisitions via horizontal transfer of a group I intron in the mitochondrial cox1 gene during evolution of the Araceae family. Mol. Biol. Evol. 16, 1155-1165.

Christensen, A. C. (2013). Plant mitochondrial genome evolution can be explained by DNA repair mechanisms. Genome Biol. Evol. 5, 1079-1086. doi: 10.1093/gbe/ evt069

De Chiara, M., Friedrich, A., Barré, B., Breitenbach, M., Schacherer, J., and Liti, G. (2020). Discordant evolution of mitochondrial and nuclear yeast genomes at population level. BMC Biol. 18:49. doi: 10.1186/s12915-020-00786-4

Dieckmann, C. L., and Gandy, B. (1987). Preferential recombination between GC clusters in yeast mitochondrial DNA. EMBO J. 6, 4197-4203.

Dimitrov, L. N., Brem, R. B., Kruglyak, L., and Gottschling, D. E. (2009). Polymorphisms in multiple genes contribute to the spontaneous mitochondrial genome instability of Saccharomyces cerevisiae S288C strains. Genetics 183, 365-383. doi: 10.1534/genetics.109.104497 contrast genomic features. The future of yeast mitogenome studies is bright, and the generated knowledge will no doubt benefit our understanding of mitogenomes much beyond the fungal kingdom.

\section{AUTHOR CONTRIBUTIONS}

WH wrote the manuscript.

\section{FUNDING}

Previous work in the lab was supported by funds from Wayne State University and the National Science Foundation (ISO1838291) to WH.

\section{ACKNOWLEDGMENTS}

I am grateful to $\mathrm{GH}$ and three reviewers for helpful comments on a previous version of this manuscript.

Dujon, B. (1989). Group I introns as mobile genetic elements: facts and mechanistic speculations-a review. Gene 82, 91-114.

Dujon, B. (2010). Yeast evolutionary genomics. Nat. Rev. Genet. 11, 512-524. doi: $10.1038 / \mathrm{nrg} 2811$

Dujon, B. (2020). Mitochondrial genetics revisited. Yeast 37, 191-205. doi: 10.1002/ yea. 3445

Dujon, B., Slonimski, P. P., and Weill, L. (1974). Mitochondrial genetics IX: a model for recombination and segregation of mitochondrial genomes in saccharomyces cerevisiae. Genetics 78, 415-437. doi: 10.1093/genetics/78.1.415

Fekete, V., Cierna, M., Poláková, S., Piskur, J., and Sulo, P. (2007). Transition of the ability to generate petites in the Saccharomyces/Kluyveromyces complex. FEMS Yeast Res. 7, 1237-1247. doi: 10.1111/j.1567-1364.2007.00287.x

Fonseca, P. L. C., De-Paula, R. B., Araújo, D. S., Tomé, L. M. R., MendesPereira, T., Rodrigues, W. F. C., et al. (2021). Global characterization of fungal mitogenomes: new insights on genomic diversity and dynamism of coding genes and accessory elements. Front. Microbiol. 12:787283. doi: 10.3389/fmicb. 2021.787283

Freel, K. C., Friedrich, A., Hou, J., and Schacherer, J. (2014). Population genomic analysis reveals highly conserved mitochondrial genomes in the yeast species Lachancea thermotolerans. Genome Biol. Evol. 6, 2586-2594. doi: 10.1093/gbe/ evu203

Fritsch, E. S., Chabbert, C. D., Klaus, B., and Steinmetz, L. M. (2014). A genomewide map of mitochondrial DNA recombination in yeast. Genetics 198, 755 771. doi: 10.1534/genetics.114.166637

Gabaldon, T., Martin, T., Marcet-Houben, M., Durrens, P., Bolotin-Fukuhara, M., Lespinet, O., et al. (2013). Comparative genomics of emerging pathogens in the Candida glabrata clade. BMC Genomics 14:623. doi: 10.1186/1471-2164-14-623

Gardner, M. J., Hall, N., Fung, E., White, O., Berriman, M., Hyman, R. W., et al. (2002). Genome sequence of the human malaria parasite Plasmodium falciparum. Nature 419, 498-511. doi: 10.1038/nature01097

Goddard, M. R., and Burt, A. (1999). Recurrent invasion and extinction of a selfish gene. Proc. Natl. Acad. Sci. U.S.A. 96, 13880-13885.

Hao, W., Richardson, A. O., Zheng, Y., and Palmer, J. D. (2010). Gorgeous mosaic of mitochondrial genes created by horizontal transfer and gene conversion. Proc. Natl. Acad. Sci. U.S.A. 107, 21576-21581. doi: 10.1073/pnas.1016295107

Hershberg, R., and Petrov, D. A. (2010). Evidence that mutation is universally biased towards AT in bacteria. PLoS Genet. 6:e1001115. doi: 10.1371/journal. pgen.1001115

Hudspeth, M. E., Vincent, R. D., Perlman, P. S., Shumard, D. S., Treisman, L. O., and Grossman, L. I. (1984). Expandable var1 gene of yeast mitochondrial DNA: 
in-frame insertions can explain the strain-specific protein size polymorphisms. Proc. Natl. Acad. Sci. U.S.A. 81, 3148-3152. doi: 10.1073/pnas.81.10.3148

Jacquier, A., and Dujon, B. (1985). An intron-encoded protein is active in a gene conversion process that spreads an intron into a mitochondrial gene. Cell 41, 383-394. doi: 10.1016/s0092-8674(85)80011-8

Jiang, H., Guan, W., Pinney, D., Wang, W., and Gu, Z. (2008). Relaxation of yeast mitochondrial functions after whole-genome duplication. Genome Res. 18, 1466-1471. doi: 10.1101/gr.074674.107

Kiktev, D. A., Sheng, Z., Lobachev, K. S., and Petes, T. D. (2018). GC content elevates mutation and recombination rates in the yeast Saccharomyces cerevisiae. Proc. Natl. Acad. Sci. U.S.A. 115, E7109-E7118. doi: 10.1073/pnas. 1807334115

Kleese, R. A., Grotbeck, R. C., and Snyder, J. R. (1972). Recombination among three mitochondrial genes in yeast (Saccharomyces cerevisiae). J. Bacteriol. 112, 1023-1025.

Koszul, R., Malpertuy, A., Frangeul, L., Bouchier, C., Wincker, P., Thierry, A., et al. (2003). The complete mitochondrial genome sequence of the pathogenic yeast Candida (Torulopsis) glabrata. FEBS Lett. 534, 39-48.

Lang, B. F., Gray, M. W., and Burger, G. (1999). Mitochondrial genome evolution and the origin of eukaryotes. Annu. Rev. Genet. 33, 351-397. doi: 10.1146/ annurev.genet.33.1.351

Lang, B. F., Jakubkova, M., Hegedusova, E., Daoud, R., Forget, L., Brejova, B., et al. (2014). Massive programmed translational jumping in mitochondria. Proc Natl Acad Sci U S A 111, 5926-5931. doi: 10.1073/pnas.1322190111

Leducq, J. B., Henault, M., Charron, G., Nielly-Thibault, L., Terrat, Y., Fiumera, H. L., et al. (2017). Mitochondrial recombination and introgression during speciation by hybridization. Mol. Biol. Evol. 34, 1947-1959. doi: 10.1093/ molbev/msx139

Ling, F., Bradshaw, E., and Yoshida, M. (2019). Prevention of mitochondrial genomic instability in yeast by the mitochondrial recombinase Mhr1. Sci. Rep. 9:5433. doi: 10.1038/s41598-019-41699-9

Ling, F., Hori, A., and Shibata, T. (2007). DNA recombination-initiation plays a role in the extremely biased inheritance of yeast [rho-] mitochondrial DNA that contains the replication origin ori5. Mol. Cell Biol. 27, 1133-1145. doi: 10.1128/MCB.00770-06

Ling, F., Hori, A., Yoshitani, A., Niu, R., Yoshida, M., and Shibata, T. (2013). Din7 and Mhr1 expression levels regulate double-strand-break-induced replication and recombination of mtDNA at ori5 in yeast. Nucleic Acids Res. 41, 5799-5816. doi: $10.1093 / \mathrm{nar} / \mathrm{gkt} 273$

Lockshon, D., Weifel, S. G., Freeman-Cook, L. L., Lorimer, H. E., Brewer, B. J., and Fangman, W. L. (1995). A role for recombination junctions in the segregation of mitochondrial DNA in yeast. Cell 81, 947-955.

Lynch, M. (2006). The origins of eukaryotic gene structure. Mol. Biol. Evol. 23, 450-468. doi: 10.1093/molbev/msj050

Lynch, M., and Conery, J. S. (2003). The origins of genome complexity. Science 302, 1401-1404. doi: 10.1126/science. 1089370

Lynch, M., Koskella, B., and Schaack, S. (2006). Mutation pressure and the evolution of organelle genomic architecture. Science 311, 1727-1730. doi: 10. 1126/science. 1118884

Lynch, M., Ung, W., Morris, K., Coffey, N., Landry, C. R., Dopman, E. B., et al. (2008). A genome-wide view of the spectrum of spontaneous mutations in yeast. Proc. Natl. Acad. Sci. U.S.A. 105, 9272-9277. doi: 10.1073/pnas.0803466105

McCutcheon, J. P., and Moran, N. A. (2010). Functional convergence in reduced genomes of bacterial symbionts spanning $200 \mathrm{My}$ of evolution. Genome Biol. Evol. 2, 708-718. doi: 10.1093/gbe/evq055

Mower, J. P., Stefanović, S., Hao, W., Gummow, J. S., Jain, K., Ahmed, D., et al. (2010). Horizontal acquisition of multiple mitochondrial genes from a parasitic plant followed by gene conversion with host mitochondrial genes. BMC Biol. 8:150. doi: 10.1186/1741-7007-8-150

Mukhopadhyay, J., and Hausner, G. (2021). Organellar introns in fungi, algae, and plants. Cells 10:2001. doi: 10.3390/cells10082001

Nguyen, D. T., Wu, B., Long, H., Zhang, N., Patterson, C., Simpson, S., et al. (2020a). Variable spontaneous mutation and loss of heterozygosity among heterozygous genomes in yeast. Mol. Biol. Evol. 37, 3118-3130. doi: 10.1093/ molbev/msaa150

Nguyen, D. T., Wu, B., Xiao, S., and Hao, W. (2020b). Evolution of a record-setting AT-rich genome: indel mutation, recombination, and substitution bias. Genome Biol. Evol. 12, 2344-2354. doi: 10.1093/gbe/evaa202
Perlman, P. S., and Butow, R. A. (1989). Mobile introns and intron-encoded proteins. Science 246, 1106-1109. doi: 10.1126/science.2479980

Pessia, E., Popa, A., Mousset, S., Rezvoy, C., Duret, L., and Marais, G. A. (2012). Evidence for widespread GC-biased gene conversion in eukaryotes. Genome Biol. Evol. 4, 675-682. doi: 10.1093/gbe/evs052

Piskur, J. (2001). Origin of the duplicated regions in the yeast genomes. Trends Genet. 17, 302-303. doi: 10.1016/s0168-9525(01)02308-3

Pramateftaki, P. V., Kouvelis, V. N., Lanaridis, P., and Typas, M. A. (2006). The mitochondrial genome of the wine yeast Hanseniaspora uvarum: a unique genome organization among yeast/fungal counterparts. FEMS Yeast Res. 6, 77-90. doi: 10.1111/j.1567-1364.2005.00018.x

Repar, J., and Warnecke, T. (2017). Mobile introns shape the genetic diversity of their host genes. Genetics 205, 1641-1648. doi: 10.1534/genetics.116.199059

Roger, A. J., Muñoz-Gómez, S. A., and Kamikawa, R. (2017). The origin and diversification of mitochondria. Curr. Biol. 27, R1177-R1192. doi: 10.1016/j. cub.2017.09.015

Sanchez-Puerta, M. V., Abbona, C. C., Zhuo, S., Tepe, E. J., Bohs, L., Olmstead, R. G., et al. (2011). Multiple recent horizontal transfers of the cox1 intron in Solanaceae and extended co-conversion of flanking exons. BMC Evol. Biol. 11:277. doi: 10.1186/1471-2148-11-277

Schikora-Tamarit, M. À, Marcet-Houben, M., Nosek, J., and Gabaldón, T. (2021). Shared evolutionary footprints suggest mitochondrial oxidative damage underlies multiple complex I losses in fungi. Open Biol. 11, 200362. doi: 10.1098/ rsob.200362

Shannon, C., Rao, A., Douglass, S., and Criddle, R. S. (1972). Recombination in yeast mitochondrial DNA. J. Supramol. Struct. 1, 145-152. doi: 10.1002/jss. 400010207

Shao, R., Kirkness, E. F., and Barker, S. C. (2009). The single mitochondrial chromosome typical of animals has evolved into 18 minichromosomes in the human body louse, Pediculus humanus. Genome Res. 19, 904-912. doi: 10.1101/ gr.083188.108

Sharp, N. P., Sandell, L., James, C. G., and Otto, S. P. (2018). The genomewide rate and spectrum of spontaneous mutations differ between haploid and diploid yeast. Proc. Natl. Acad. Sci. U.S.A. 115, E5046-E5055. doi: 10.1073/pnas. 1801040115

Shtolz, N., and Mishmar, D. (2019). The mitochondrial genome-on selective constraints and signatures at the organism, cell, and single mitochondrion levels. Front. Ecol. Evol. 7:342. doi: 10.3389/fevo.2019.00342

Sloan, D. B., Alverson, A. J., Chuckalovcak, J. P., Wu, M., McCauley, D. E., Palmer, J. D., et al. (2012). Rapid evolution of enormous, multichromosomal genomes in flowering plant mitochondria with exceptionally high mutation rates. PLoS Biol. 10:e1001241. doi: 10.1371/journal.pbio.1001241

Smith, D. R. (2015). The past, present and future of mitochondrial genomics: have we sequenced enough mtDNAs? Brief. Funct. Genomics 15, 47-54. doi: 10.1093/bfgp/elv027

Smith, D. R., Burki, F., Yamada, T., Grimwood, J., Grigoriev, I. V., Van Etten, J. L., et al. (2011). The GC-rich mitochondrial and plastid genomes of the green alga Coccomyxa give insight into the evolution of organelle DNA nucleotide landscape. PLoS One 6:e23624. doi: 10.1371/journal.pone.002 3624

Smith, D. R., Kayal, E., Yanagihara, A. A., Collins, A. G., Pirro, S., and Keeling, P. J. (2012). First complete mitochondrial genome sequence from a box jellyfish reveals a highly fragmented linear architecture and insights into telomere evolution. Genome Biol. Evol. 4, 52-58. doi: 10.1093/gbe/evr127

Strausberg, R. L., and Butow, R. A. (1981). Gene conversion at the var1 locus on yeast mitochondrial DNA. Proc. Natl. Acad. Sci. U.S.A. 78, 494-498.

Strope, P. K., Skelly, D. A., Kozmin, S. G., Mahadevan, G., Stone, E. A., Magwene, P. M., et al. (2015). The 100-genomes strains, an S. cerevisiae resource that illuminates its natural phenotypic and genotypic variation and emergence as an opportunistic pathogen. Genome Res. 25, 762-774. doi: 10.1101/gr.18553 8.114

Su, H. J., Barkman, T. J., Hao, W., Jones, S. S., Naumann, J., Skippington, E., et al. (2019). Novel genetic code and record-setting AT-richness in the highly reduced plastid genome of the holoparasitic plant Balanophora. Proc. Natl. Acad. Sci. U.S.A. 116, 934-943. doi: 10.1073/pnas.1816822116

Weiller, G. F., Bruckner, H., Kim, S. H., Pratje, E., and Schweyen, R. J. (1991). A $\mathrm{GC}$ cluster repeat is a hotspot for mit- macro-deletions in yeast mitochondrial DNA. Mol Gen Genet 226, 233-240. 
Weiller, G., Schueller, C. M., and Schweyen, R. J. (1989). Putative target sites for mobile $\mathrm{G}+\mathrm{C}$ rich clusters in yeast mitochondrial DNA: single elements and tandem arrays. Mol. Gen. Genet. 218, 272-283.

Whitney, K. D., and Garland, T. Jr. (2010). Did genetic drift drive increases in genome complexity? PLoS Genet. 6:e1001080. doi: 10.1371/journal.pgen. 1001080

Whittaker, P. A., Hammond, R. C., and Luha, A. A. (1972). Mechanism of mitochondrial mutation in yeast. Nat. New Biol. 238, 266-268. doi: 10.1038/ newbio238266a0

Wilkie, D., and Thomas, D. Y. (1973). Mitochondrial genetic analysis by zygote cell lineages in Saccharomyces cerevisiae. Genetics 73, 367-377. doi: 10.1093/ genetics/73.3.367

Wolters, J. F., Charron, G., Gaspary, A., Landry, C. R., Fiumera, A. C., and Fiumera, H. L. (2018). Mitochondrial recombination reveals mito-mito epistasis in yeast. Genetics 209, 307-319. doi: 10.1534/genetics.117.300660

$\mathrm{Wu}, \mathrm{B}$., and Hao, W. (2014). Horizontal transfer and gene conversion as an important driving force in shaping the landscape of mitochondrial introns. G3 $4,605-612$.

Wu, B., and Hao, W. (2015). A dynamic mobile DNA family in the yeast mitochondrial genome. G3 5, 1273-1282. doi: 10.1534/g3.115.017822

$\mathrm{Wu}, \mathrm{B}$., and Hao, W. (2019). Mitochondrial-encoded endonucleases drive recombination of protein-coding genes in yeast. Environ. Microbiol. 21, 42334240. doi: 10.1111/1462-2920.14783

Wu, B., Buljic, A., and Hao, W. (2015). Extensive horizontal transfer and homologous recombination generate highly chimeric mitochondrial genomes in yeast. Mol. Biol. Evol. 32, 2559-2570. doi: 10.1093/molbev/msv127
Xiao, S., Nguyen, D. T., Wu, B., and Hao, W. (2017). Genetic drift and indel mutation in the evolution of yeast mitochondrial genome size. Genome Biol. Evol. 9, 3088-3099. doi: 10.1093/gbe/evx232

Yin, S., Heckman, J., and RajBhandary, U. L. (1981). Highly conserved GCrich palindromic DNA sequences flank tRNA genes in Neurospora crassa mitochondria. Cell 26, 325-332. doi: 10.1016/0092-8674(81)90201-4

Zhu, Y. O., Siegal, M. L., Hall, D. W., and Petrov, D. A. (2014). Precise estimates of mutation rate and spectrum in yeast. Proc. Natl. Acad. Sci. U.S.A. 111, E2310-E2318. doi: 10.1073/pnas.1323011111

Conflict of Interest: The author declares that the research was conducted in the absence of any commercial or financial relationships that could be construed as a potential conflict of interest.

Publisher's Note: All claims expressed in this article are solely those of the authors and do not necessarily represent those of their affiliated organizations, or those of the publisher, the editors and the reviewers. Any product that may be evaluated in this article, or claim that may be made by its manufacturer, is not guaranteed or endorsed by the publisher.

Copyright (c) $2022 \mathrm{Hao}$. This is an open-access article distributed under the terms of the Creative Commons Attribution License (CC BY). The use, distribution or reproduction in other forums is permitted, provided the original author(s) and the copyright owner(s) are credited and that the original publication in this journal is cited, in accordance with accepted academic practice. No use, distribution or reproduction is permitted which does not comply with these terms. 